\title{
Effect of solvent topography and steric hindrance on crystal morphology
}

\author{
Charles Acquah $^{\mathrm{a}}$, Matthew Cagnetta ${ }^{\mathrm{a}}$, Luke E. K. Achenie ${ }^{\mathrm{b}}$, Steven L. Suib ${ }^{\mathrm{c}}$, and \\ Arunprakash T. Karunanithi ${ }^{\mathrm{d}^{*}}$
}

${ }^{a}$ Department of Chemical Engineering, University of Connecticut, CT, USA.

${ }^{b}$ Department of Chemical Engineering, Virginia Polytechnic Institute and State University, Blacksburg, VA, USA.

${ }^{c}$ Department of Chemistry, University of Connecticut, Storrs, CT, USA.

${ }^{d}$ Center for Sustainable Infrastructure Systems, University of Colorado Denver, Denver, CO, USA. 
Table S1. Selected solvents

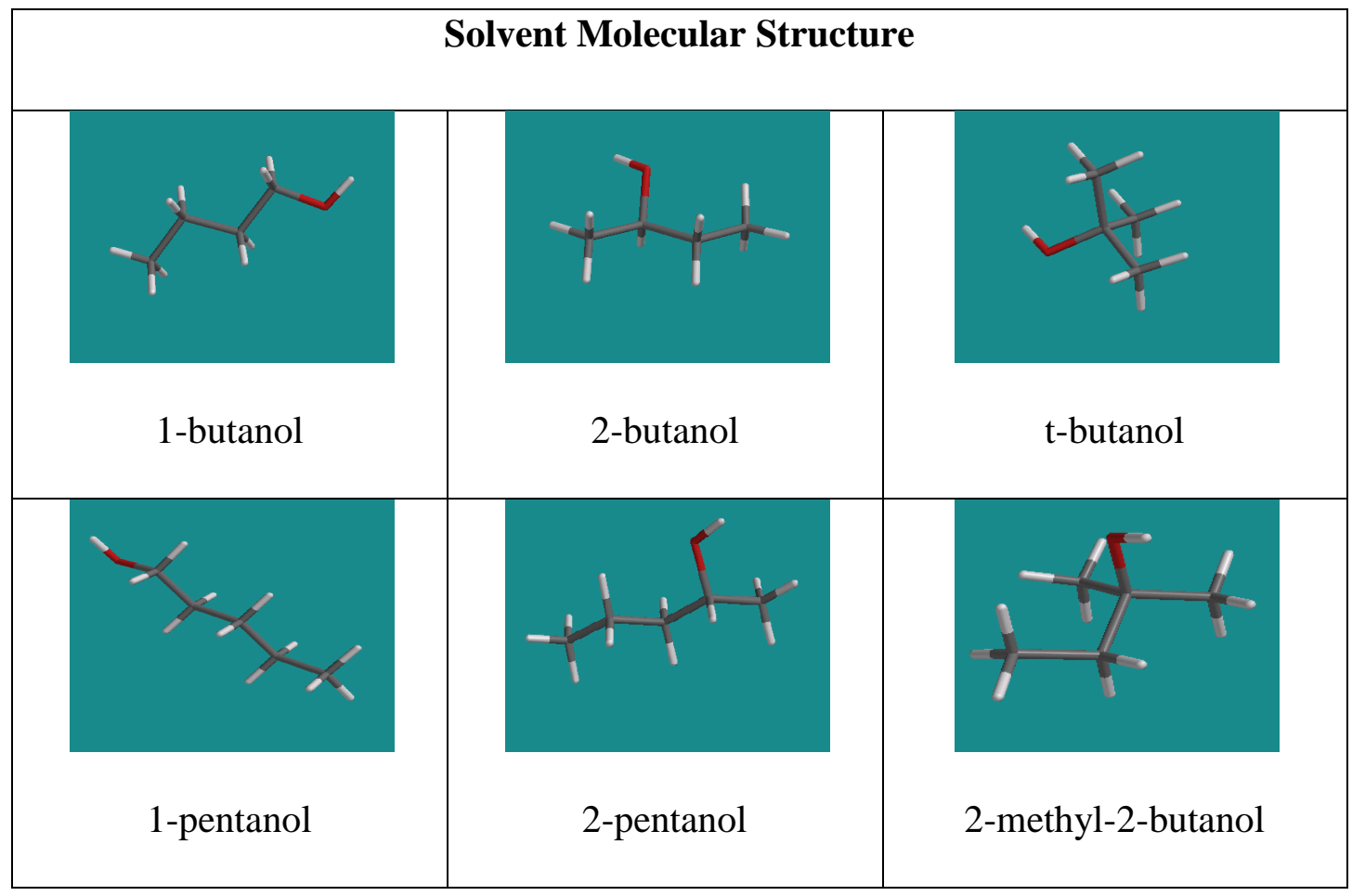


Table S2. Properties of butyl- isomers.

\begin{tabular}{|l|c|c|}
\hline Solvent & $\boldsymbol{\delta}_{\boldsymbol{H}}$ & $\boldsymbol{\alpha}$ \\
\hline 1-butanol & 15.8 & 0.84 \\
\hline 2-butanol & 14.5 & 0.69 \\
\hline $\mathrm{t}$-butanol & 14.7 & 0.42 \\
\hline $\begin{array}{l}\delta_{H}=\text { Hansen hydrogen bonding solubility parameter, }\left[\mathrm{MPa}^{1 / 2}\right] \\
\alpha=\text { Kamlet-Taft parameter }\end{array}$ \\
\hline
\end{tabular}

Table S3. Properties of pentyl- isomers.

\begin{tabular}{|l|c|c|}
\hline Solvent & $\boldsymbol{\delta}_{\boldsymbol{H}}$ & $\alpha$ \\
\hline 1-pentanol & 13.9 & 0.84 \\
\hline 2-pentanol & 13.3 & 0.84 \\
\hline 2-methyl-2-butanol & 13.3 & 0.28 \\
\hline
\end{tabular}


Table S4. Aspect ratio for various solvent-solute systems

\begin{tabular}{|c|c|c|c|}
\hline Solute & Solvent & Aspect Ratio & Valence \\
\hline \multirow[t]{6}{*}{$\mathbf{S A}$} & 1-butanol & $1.54 \pm 0.46$ & 1 \\
\hline & 2-butanol & $1.66 \pm 0.35$ & 2 \\
\hline & t-butanol & $3.62 \pm 0.50$ & 3 \\
\hline & 1-pentanol & $1.69 \pm 0.37$ & 1 \\
\hline & 2-pentanol & $2.35 \pm 0.39$ & 2 \\
\hline & 2-methyl-2-butanol & $3.69 \pm 0.54$ & 3 \\
\hline \multirow[t]{6}{*}{ PA } & 1-butanol & $1.55 \pm 0.26$ & 1 \\
\hline & 2-butanol & $1.85 \pm 0.45$ & 2 \\
\hline & t-butanol & $2.87 \pm 0.55$ & 3 \\
\hline & 1-pentanol & $1.80 \pm 0.38$ & 1 \\
\hline & 2-pentanol & $1.96 \pm 0.43$ & 2 \\
\hline & 2-methyl-2-butanol & $3.85 \pm 0.83$ & 3 \\
\hline \multirow[t]{6}{*}{ ASA } & 1-butanol & $1.95 \pm 0.33$ & 1 \\
\hline & 2-butanol & $3.35 \pm 0.42$ & 2 \\
\hline & t-butanol & $3.97 \pm 0.55$ & 3 \\
\hline & 1-pentanol & $2.41 \pm 0.39$ & 1 \\
\hline & 2-pentanol & $3.95 \pm 0.70$ & 2 \\
\hline & 2-methyl-2-butanol & $4.82 \pm 0.70$ & 3 \\
\hline
\end{tabular}


Table S5. Statistical analysis for solvent-solute systems

\begin{tabular}{|c|c|c|c|c|}
\hline Solute & Pairwise Tests & $t$-critical & $t$-value & conclusion \\
\hline \multirow{8}{*}{ ASA } & Butyl isomers & & & \\
\hline & $1^{\circ}$ vs $2^{\circ}$ & 2.048 & 10.11 & significant \\
\hline & $2^{\circ}$ vs $3^{\circ}$ & 2.048 & 3.44 & significant \\
\hline & $1^{\circ}$ vs $3^{\circ}$ & 2.048 & 12.15 & significant \\
\hline & Pentyl isomers & & & \\
\hline & $1^{\circ}$ vs $2^{\circ}$ & 2.048 & 7.49 & significant \\
\hline & $2^{\circ}$ vs $3^{\circ}$ & 2.048 & 3.40 & significant \\
\hline & $1^{\circ}$ vs $3^{\circ}$ & 2.048 & 11.63 & significant \\
\hline \multirow{8}{*}{ PA } & Butyl isomers & & & \\
\hline & $1^{\circ}$ vs $2^{\circ}$ & 2.048 & 2.22 & significant \\
\hline & $2^{\circ}$ vs $3^{\circ}$ & 2.048 & 5.52 & significant \\
\hline & $1^{\circ}$ vs $3^{\circ}$ & 2.048 & 8.36 & significant \\
\hline & Pentyl isomers & & & \\
\hline & $1^{\circ}$ vs $2^{\circ}$ & 2.048 & 1.09 & not significant \\
\hline & $2^{\circ}$ vs $3^{\circ}$ & 2.048 & 7.88 & significant \\
\hline & $1^{\circ}$ vs $3^{\circ}$ & 2.048 & 8.74 & significant \\
\hline \multirow{5}{*}{$\mathbf{S A}$} & Butyl isomers & & & \\
\hline & $1^{\circ}$ vs $2^{\circ}$ & 2.048 & 1.07 & not significant \\
\hline & $2^{\circ}$ vs $3^{\circ}$ & 2.048 & 12.34 & significant \\
\hline & $1^{\circ}$ vs $3^{\circ}$ & 2.048 & 13.89 & significant \\
\hline & Pentyl isomers & & & \\
\hline
\end{tabular}




\begin{tabular}{|c|c|c|c|c|}
\hline & $1^{\circ}$ vs $2^{\circ}$ & 2.048 & 4.69 & significant \\
\cline { 2 - 5 } & $2^{\circ}$ vs $3^{\circ}$ & 2.048 & 7.78 & significant \\
\cline { 2 - 5 } & $1^{\circ}$ vs $3^{\circ}$ & 2.048 & 11.80 & significant \\
\hline
\end{tabular}

